\title{
Peak systolic velocity of tricuspid annulus is inferior to tricuspid annular plane systolic excursion for 30 days prediction of adverse outcome in acute pulmonary embolism
}

\author{
Katarzyna Kurnicka ${ }^{1}$, Barbara Lichodziejewska ${ }^{1}$, Michał Ciurzyński ${ }^{1}$, \\ Maciej Kostrubiec ${ }^{1}$, Sylwia Goliszek ${ }^{1}$, Olga Zdończyk ${ }^{1}$, \\ Olga Dzikowska-Diduch ${ }^{1}$, Piotr Palczewski ${ }^{2}$, Marta Skowrońska ${ }^{1}$, \\ Marcin Koć ${ }^{1}$, Katarzyna Grudzka ${ }^{1}$, Piotr Pruszczyk ${ }^{1}$
}

${ }^{1}$ Department of Internal Medicine and Cardiology, Medical University of Warsaw, Poland ${ }^{2} 1^{\text {st }}$ Department of Radiology, Medical University of Warsaw, Poland

\begin{abstract}
Background: Tricuspid annular plane systolic excursion (TAPSE) is an established index of right ventricular $(R V)$ systolic function and a significant predictor in normotensive patients with pulmonary embolism (PE). Recently, Doppler tissue imaging-derived tricuspid annular systolic velocity (TV S'), a modern parameter of RV function was reported to be useful in the diagnosis and prognosis of a broad spectrum of heart diseases. Therefore, herein, is an analysis of the prognostic value of both parameters in normotensive PE patients.

Methods: One hundred and thirty nine consecutive PE patients (76 female, age $56.4 \pm 19.5$ years) were included in this study. All patients were initially anticoagulated. Transthoracic echocardiography was performed on admission. The study endpoint (SE) was defined as PE-related 30-day mortality and/ or need for rescue thrombolysis.

Results. Seven (5\%) patients who met the criteria for SE presented more severe RV dysfunction at echocardiography. Univariable Cox regression analysis showed that $R V / L V$ ratio predicted $S E$ with hazard risk (HR) 10.6 (1.4-80.0; $p=0.02)$; TAPSE and TV S' showed HR 0.77 (0.67-0.89), $p<0.001$, and $0.71(0.52-0.97), p=0.03$, respectively. Area under the curve for TAPSE in the prediction of SE was 0.881; 95\% CI 0.812-0.932, $p=0.0001$, for TV S' was 0.751; 95\% CI 0.670-0.820, $p=0.001$. Multivariable analysis showed that the optimal prediction model included TAPSE and systolic blood pressure (SBP showed HR 0.89 95\% CI 0.83-0.95, p < 0.001 and TAPSE HR 0.67, 95\% CI 0.52-0.87, $p<0.03)$. Kaplan-Meier analysis showed that initially PE patients with TAPSE $\geq 18 \mathrm{~mm}$ had a much more favorable prognosis that patients with TAPSE $<18 \mathrm{~mm}(p<0.01)$, while analysis of S' was only of borderline statistical significance.

Conclusions: It seems that TV S' is inferior to TAPSE for 30 day prediction of adverse outcome in acute pulmonary embolism. (Cardiol J 2020; 27, 5: 558-565)

Key words: transthoracic echocardiography, right ventricular function, tricuspid valve, Doppler tissue imaging, prognosis, pulmonary embolism
\end{abstract}

Address for correspondence: Dr n. med. Katarzyna Kurnicka, Department of Internal Medicine and Cardiology, Medical University of Warsaw, ul. Lindleya 4, 02-005 Warszaawa, Poland, tel: +48 22 5021144, fax: +48 22 5022142, e-mail: kkurnicka@yahoo.pl Received: 15.07.2018 Accepted: 8.11.2018 


\section{Introduction}

Normotensive patients with acute pulmonary embolism (PE) include not only subjects with a benign clinical course, but also patients with an increased risk of PE-related mortality. Intermediate-high-risk $\mathrm{PE}$ patients require close monitoring to detect hemodynamic decompensation and to consider rescue reperfusion therapy [1].

Right ventricular dysfunction (RVD) detected at computed tomography or in transthoracic echocardiography (TTE) indicates an increased risk of a complicated clinical course, including early mortality. The assessment of right ventricle (RV) function is recommended in the current 2014 guidelines of the European Society of Cardiology (ESC) for risk stratification of normotensive PE patients [1, 2]. However, despite accumulating evidence there is generally no accepted echocardiographic definition of RVD proposed for risk stratification in normotensive PE patients. The criteria used to define RVD vary between studies and have included RV dilatation, an increased $\mathrm{RV} / \mathrm{LV}$ diameter ratio, hypokinesis of the free $\mathrm{RV}$ wall, an increased velocity of the tricuspid regurgitation jet [1-4]. As shown previously, that in initially normotensive PE patients tricuspid annular plane systolic excursion (TAPSE), the only independent echocardiographic outcome predictor among a wide set of echocardiographic indices [5], and others subsequently reported similar results [6].

Doppler tissue imaging (DTI) has become a widely available technique, which plays an important role in the diagnosis and prognosis of a broad spectrum of heart diseases [7-9]. It is a simple, reproducible diagnostic method with a good ability to detect RV dysfunction [10]. DTI-derived tricuspid lateral annular systolic velocity (TV S') correlates well with other parameters of global RV systolic function [11, 12]. It was reported that the assessment of RV myocardial S' velocity by DTI may be adequate to confirm RVD in patients with PE $[13,14]$. DTI can be also used to monitor RV function and filling pressure in $\mathrm{PE}$ patients [15].

Although there are some reports suggesting a prognostic value of TV S' in patients with acute $\mathrm{PE}[16,17]$, no direct comparison between TAPSE and tricuspid annular systolic velocity (TV S') is available. Therefore, the prognostic value of both parameters in normotensive PE patients was analyzed.

\section{Methods}

\section{Patients and management of acute PE}

The study group comprised of consecutive patients with symptomatic PE managed in the documented department. All cases were hemodynamically stable at admission, with systemic systolic blood pressure (SBP) exceeding $90 \mathrm{mmHg}$, and no signs of peripheral hypoperfusion. Pulmonary embolism was confirmed by contrast-enhanced multi-detector computed tomography when thromboemboli were visualized at least at the level of segmental pulmonary arteries. Acute PE was diagnosed when symptoms of $\mathrm{PE}$ had been present for no longer than 14 days before the diagnosis.

All patients were initially anticoagulated with body mass adjusted low molecular weight heparin or activated partial thromboplastin time adjusted unfractionated heparin infusion. In the case of clinical deterioration, urgent rescue thrombolysis (rtPA $0.6 \mathrm{mg} / \mathrm{kg}$ body weight, $\max 50 \mathrm{mg}$ i.v.) was performed. Hemodynamic deterioration was defined by systemic hypotension $<90 \mathrm{mmHg}$ with signs of peripheral hypoperfusion, with tachycardia exceeding $110 \mathrm{bpm}$. A decision to escalate treatment was facilitated by significant dyspnea. Oral anticoagulation preferably with non-vitamin $\mathrm{K}$ antagonist oral anticoagulants or with international normalized ratio adjusted vitamin $\mathrm{K}$ antagonists were started according to the decision of the managing physician.

Comorbidities were defined as presence of chronic obstructive pulmonary disease (COPD), chronic heart failure (CHF) or active cancer.

Patients not included were those with severe tricuspid regurgitation, a history of RV myocardial infarction, after tricuspid valve replacement, or those with confirmed chronic thromboembolic pulmonary hypertension.

\section{Echocardiography}

Standardized transthoracic echocardiography focused on the assessment of RV function was performed using Philips iE33 (Philips Medical Systems, Andover, Massachusetts) and Philips Epiq 7 systems (Philips Medical Systems, Best, the Netherlands) according to the previously described protocol [18], as soon as possible after admission.

Briefly, left ventricle and right ventricle diameters were measured in the apical four chamber view (LV4C, RV4C, respectively) at the level of the mitral and tricuspid valve tips at end-diastole (defined by the ECG R wave). The presence of the McConnell sign was assessed [19]. TAPSE was measured using M-mode presentation in the 
apical 4-chamber view, with the cursor exactly aligned along the direction of the tricuspid lateral annulus [11].

Peak systolic velocity of the lateral part of the tricuspid annulus - TV S' was measured using DTI. Sample volume of pulsed DTI was placed in the tricuspid annulus or in the middle of the basal segment of the RV free wall. To achieve a high quality of recording optimal gain was used. The TV S' velocity was defined as the highest systolic velocity, without over gaining the Doppler envelope $[10,11]$. The isovolumic contraction velocities were excluded from the TV S' measurement.

In the parasternal short axis view, flattening of the interventricular septum was assessed qualitatively, and the acceleration time (AcT) of pulmonary ejection was measured in the right ventricle outflow tract, proximally to the pulmonary valve (PW Doppler).

Tricuspid regurgitation peak systolic gradient (TRPG) was calculated by the simplified Bernoulli formula using tricuspid regurgitant flow peak velocity (Doppler continous wave). The examination was completed by the measurement of the inferior vena cava (IVC) at late expiration.

Left ventricular ejection fraction (LVEF) was measured with the modified biplane Simpson method [11]. Doppler measurements reflected an average of three cardiac cycles. The inter- and intra-observer agreement for echocardiographic parameters were previously published [18].

Examinations were digitally recorded and interpreted by experienced sonographers according to a standardized protocol following recommendations of the European Association of Cardiovascular Imaging [11].

In 117 patients TTE was performed within the first $24 \mathrm{~h}$ after admission, while in 22 patients, this examination was performed between 24 and $72 \mathrm{~h}$ after admission.

\section{Study endpoint}

The study endpoint was a combination of 30-day PE-related mortality or need for rescue thrombolysis or both in patients with hemodynamic deterioration.

\section{Statistical analysis}

Data characterized by a normal distribution are expressed as mean followed by standard deviation. Parameters without such a distribution are expressed as median with range. The Student t-test or Mann-Whitney U-test was used for comparisons between the two groups. The chi-square/ chi-squared test was used to compare discrete variables (with the Yates correction when needed). Receiver operating characteristics (ROC) analysis was performed and the area under the curve (AUC) was determined to test the performance of selected echocardiographic parameters with regard to the prediction of serious adverse event (SAE). Youden's index quantification was used to identify optimal cohort-specific cut-off values. The impact of TAPSE and S' on study end points was evaluated using univariable Cox proportional-hazards regression. Hazard risk (HR) and corresponding 95\% confidence intervals (CI) were calculated. Kaplan-Meier analysis was used to investigate cumulative 30-day event free survival rate. Forward stepwise selection with a 0.1 level for staying in the model was used to identify significant predictors in multivariable analysis. Areas under ROC curves were compared pair-wise. Sensitivity, specificity, negative predictive values (NPV), positive predictive values (PPV), and the corresponding 95\% CI were calculated for TAPSE and TV S'. All tests were two-sided. Data were considered significant at $\mathrm{p}<0.05$. STATISTICA data analysis software system (StatSoft, Inc. 2011, version 10, www.statsoft.com) and MedCalc ${ }^{\circledR}$ software (version 11.0.0.0) were used for statistical calculations. This observational study was approved by the local Ethics Committee.

\section{Results}

Patients characteristics and clinical course

The study included 139 consecutive patients with PE (63 males, 76 females, age $56.4 \pm 19.5$ years), normotensive at admission. The study outcome defined as 30-day PE-related mortality and/ /or need for rescue thrombolysis in patients with hemodynamic deterioration was observed in $7(5 \%)$ of the patients studied. Despite anticoagulation $6(4.3 \%)$ patients experienced hemodynamic collapse and underwent rescue thrombolysis, 4 of them survived. 30-day PE-related mortality was $2.2 \%$ (3 patients), and all-cause mortality was $2.9 \%$ (4 patients). The one none$\mathrm{PE}$ related death occurred in a 92 -year-old patient with metastatic cancer. Initial systemic SBP was significantly lower in patients who experienced serious adverse events $(\mathrm{SAE}(+))$ than in patients with an uncomplicated clinical course (SAE (-)). There was no difference in age and heart rate between SAE (+) and SAE (-) groups. Clinical characteristics of patients studied are presented in Table 1. 
Table 1. Clinical characteristics of 139 normotensive patients with pulmonary embolism (PE).

\begin{tabular}{lcccc}
\hline Parameter & $\begin{array}{c}\text { All patients } \\
(\mathbf{n}=\mathbf{1 3 9 )}\end{array}$ & $\begin{array}{c}\text { SAE (+) } \\
(\mathbf{n}=\mathbf{7})\end{array}$ & $\begin{array}{c}\text { SAE (-) } \\
\text { (n= 132) }\end{array}$ & P \\
\hline Female/male & $76 / 63$ & $5 / 2$ & $71 / 61$ & 0.9 \\
Age [years] & $56.4 \pm 19.5$ & $61.6 \pm 19.9$ & $56.1 \pm 19.6$ & 0.5 \\
Systemic systolic BP [mmHg] & $130 \pm 21.4$ & $100 \pm 11.5$ & $131.6 \pm 20.5$ & $\mathbf{0 . 0 0 1}$ \\
Heat rate [1/s] & $90.6 \pm 18.7$ & $106.3 \pm 22.7$ & $89.8 \pm 18.2$ & 0.1 \\
Comorbidities (COPD, CHF, neoplasm) [\%] & 9 & $1(16.7)$ & $26(19.5)$ & 0.8 \\
Rescue thrombolysis [\%] & $6(4.3)$ & $6(85.7)$ & 0 & - \\
PE-related death/all cause death & $3 / 4$ & $3 / 0$ & $0 / 1$ & - \\
\hline
\end{tabular}

Data are expressed as mean \pm standard deviation, median and range, or percentage. $\mathrm{BP}$ - blood pressure; CHF — chronic heart failure; COPD - chronic obstructive pulmonary disease; SAE — serious adverse event

Table 2. Echocardiographic characteristics of 139 initially normotensive pulmonary embolism patients.

\begin{tabular}{lcccc}
\hline Parameter & $\begin{array}{c}\text { All patients } \\
(\mathbf{n}=\mathbf{1 3 9 )}\end{array}$ & $\begin{array}{c}\text { SAE (+) } \\
(\mathbf{n}=\mathbf{7})\end{array}$ & $\begin{array}{c}\text { SAE (-) } \\
(\mathbf{n}=\mathbf{1 3 2})\end{array}$ \\
\hline RV4C [mm] & $38.8 \pm 7.7$ & $43.3 \pm 7.5$ & $38.6 \pm 7.7$ & 0.2 \\
LV4C [mm] & $40.5 \pm 6.4$ & $32.5(26-54)$ & $40.7 \pm 6.0$ & 0.35 \\
RV/LV4C & $1.0 \pm 0.3$ & $1.3 \pm 0.4$ & $1.0 \pm 0.3$ & 0.12 \\
AcT [ms] & $80(37-166)$ & $61.3 \pm 14.6$ & $81(42-165)$ & $<\mathbf{0 . 0 1 6}$ \\
TRPG [mmHg] & $33(10-106)$ & $46.5 \pm 7.2$ & $32(15-57)$ & $\mathbf{0 . 0 1}$ \\
TAPSE [mm] & $20 \pm 5$ & $14 \pm 4$ & $21 \pm 5$ & $<\mathbf{0 . 0 0 1}$ \\
TV S' [cm/s] & $13.0(6.3-25.7)$ & $10.2(6.3-12.3)$ & $13.9(6.6-25.7)$ & $\mathbf{0 . 0 3}$ \\
IVS flattening [\%] & $39(28)$ & $4(57)$ & $35(26.5)$ & 0.4 \\
McConnell sign [\%] & $39(28)$ & $4(57)$ & $35(26.5)$ & 0.4 \\
IVC [mm] & $14(5-30)$ & $19.2 \pm 4.0$ & $14(5-30)$ & $\mathbf{0 . 0 0 5}$ \\
LVEF [\%] & $59.5 \pm 6.2$ & $55(30-60)$ & $60.4 \pm 5.6$ & 0.1 \\
\hline
\end{tabular}

Data are expressed as mean \pm standard deviation, median and range or percentage. RV4C — right ventricular dimension in apical four chamber view; LV4C - left ventricular dimension in apical four chamber view, RV/LV 4C - right ventricle to left ventricle ratio in the apical four chamber view; AcT — pulmonary ejection acceleration time, TRPG — tricuspid regurgitation peak gradient; TAPSE — tricuspid annular systolic plane excursion; TV S' - peak systolic velocity of lateral part of tricuspid annulus; IVS — interventricular septum; IVC — inferior vena cava; LVEF — left ventricular ejection fraction; SAE - serious adverse event

\section{Echocardiography}

Echocardiographic data of the group studied are included in Table 2. Patients with a complicated clinical outcome presented more pronounced echocardiographic signs of RVD. Mean values of TAPSE and TV S' (Figs. 1,2) and AcT were significantly lower, while TRPG and IVC were significantly higher in the SAE $(+)$ group when compared with the SAE (-) group.

The McConnell sign and flattening of IVS were more frequent in SAE $(+)$ patients, but without statistical significance. There were no differences in RV4C dimensions, RV/LV ratio, and LVEF between SAE (+) and SAE (-) groups.

\section{Echocardiographic predictors of clinical endpoint}

Univariable Cox proportional-hazards regression analysis showed that several echocardiographic parameters significantly predicted a complicated clinical outcome. Importantly, TAPSE and TV S' showed similar HRs, HR 0.77 (0.67-0.89), $\mathrm{p}<0.001$, and $0.71(0.52-0.97), \mathrm{p}=0.03$, respectively (Table 3 ).

\section{ROC curve analysis}

ROC analysis showed that the AUC for TAPSE in the prediction of a complicated clinical course was $0.881,95 \%$ CI $0.812-0.932, \mathrm{p}=0.0001$, mean- 


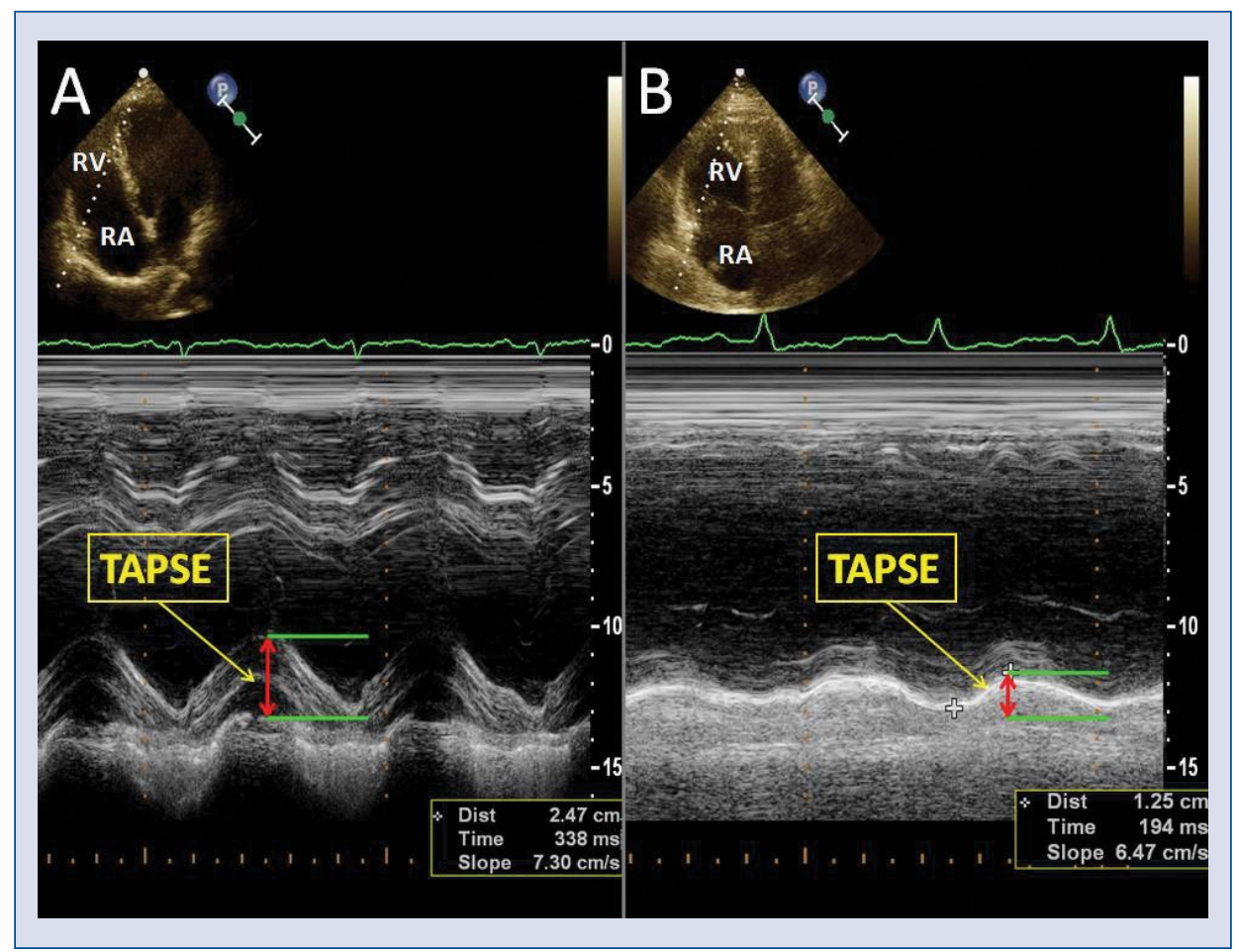

Figure 1. M-mode presentation of tricuspid annular plane systolic excursion (TAPSE) in two initially normotensive patients with acute pulmonary embolism; A. Patient with a benign clinical course (distance $25 \mathrm{~mm}$ - red double-headed arrow); B. Patient with a complicated clinical outcome and decreased annular movement (distance $12.5 \mathrm{~mm}$ - red double-headed arrow); RA — right atrium; RV — right ventricle.

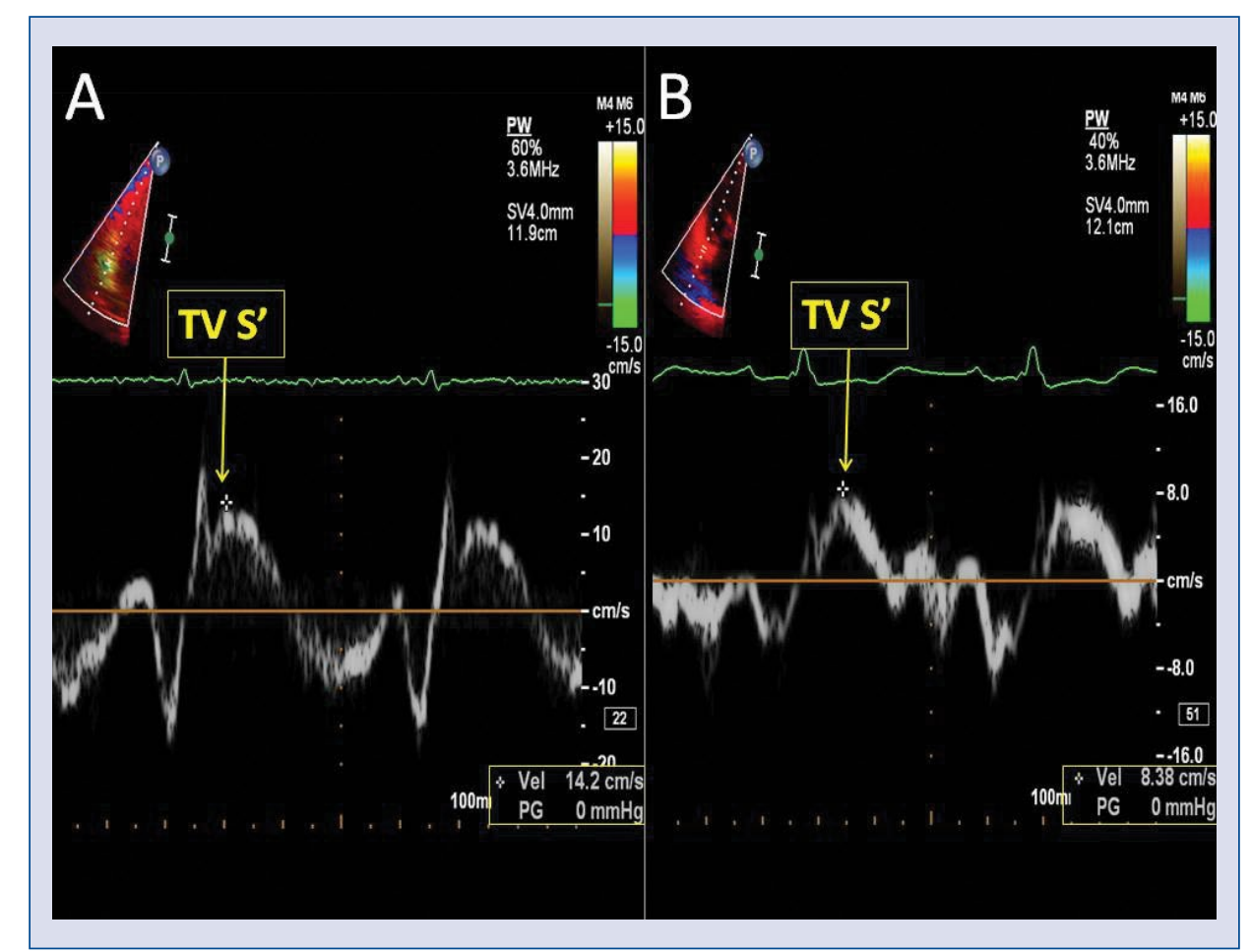

Figure 2. Tissue Doppler of the tricuspid annulus in two initially normotensive patients with acute pulmonary embolism; A. Patient with a benign clinical course (TV S' $14.2 \mathrm{~cm} / \mathrm{s}$ - yellow arrow); B. Patient with a complicated clinical outcome and decreased annular velocity (TV S' $8.4 \mathrm{~cm} / \mathrm{s}$ - yellow arrow); TV $\mathrm{S}^{\prime}$ - peak systolic velocity of lateral part of tricuspid annulus. 
Table 3. Univariable echocardiographic predictors of pulmonary embolism-related mortality or rescue thrombolysis in 139 initially normotensive patients.

\begin{tabular}{lccc}
\hline Parameter & HR & $95 \% \mathrm{Cl}$ & $\mathbf{P}$ \\
\hline TAPSE $[\mathrm{mm}]$ & 0.77 & $0.67-0.89$ & $<\mathbf{0 . 0 0 1}$ \\
TV S' $[\mathrm{cm} / \mathrm{s}]$ & 0.71 & $0.52-0.97$ & $\mathbf{0 . 0 3}$ \\
TV S' $\leq 12.3[\mathrm{~cm} / \mathrm{s}]$ & 4.3 & $0.8-22.3$ & $\mathbf{0 . 0 8}$ \\
AcT $[\mathrm{ms}]$ & 0.96 & $0.91-1.00$ & 0.06 \\
TRPG $[\mathrm{mmHg}]$ & 1.03 & $0.99-1.07$ & 0.14 \\
IVC $[\mathrm{mm}]$ & 0.14 & $0.99-1.07$ & 0.057 \\
RV/LV & 10.6 & $1.4-80.0$ & 0.02 \\
\hline
\end{tabular}

TVS $^{\prime}<12.3 \mathrm{~cm} / \mathrm{s}$ was defined using receiver operating characteristics analysis. TAPSE - tricuspid annular systolic plane excursion; TV S'- peak systolic velocity of lateral part of tricuspid annulus; AcT - pulmonary ejection acceleration time; TRPG - tricuspid regurgitation peak gradient; IVC — inferior vena cava; RV/LV — right ventricle to left ventricle ratio; $\mathrm{OR}$ - odds ratio; $\mathrm{Cl}$ - confidence interval

Table 4. Sensitivity and specificity of receiver operating characteristics derived cut-offs of tricuspid annular systolic plane excursion (TAPSE) and peak systolic velocity of lateral part of tricuspid annulus (TV $S^{\prime}$ ) in serious adverse event prediction.

\begin{tabular}{lcccc}
\hline & Sensitivity & Specificity & NPV & PPV \\
\hline $\begin{array}{l}\text { TAPSE } \\
\leq 18[\mathrm{~mm}]\end{array}$ & $100 \%$ & $68 \%$ & $100 \%$ & $16 \%$ \\
$\begin{array}{l}\text { TV S } \\
\leq 12.3[\mathrm{~cm} / \mathrm{s}]\end{array}$ & $86 \%$ & $60 \%$ & $99 \%$ & $10 \%$ \\
\hline
\end{tabular}

NPV — negative predictive value; PPV — positive predictive value

while for TV S' AUC $0.751 ; 95 \%$ CI $0.670-0.820$, $\mathrm{p}=0.001$; Fig. 3 ).

Importantly, a direct comparison showed that AUC for TAPSE and for TV S' did not differ significantly. Using the Youden's index, a cut-off point for TAPSE at $18 \mathrm{~mm}$ and for TV S' at $12.3 \mathrm{~cm} / \mathrm{s}$ were identified as optimal values for SAE prediction. When cut off values of TAPSE and TVS' defined according to ROC analysis were used in hazard risk analysis TAPSE $<18$ showed HR for study end point of $16.3(2.0-135.3, \mathrm{p}=0.01)$, while TV'S $<12.3 \mathrm{~cm} / \mathrm{s}$ was only of borderline significance HR $4.3(0.8-22.2, \mathrm{p}=0.08)$.

Table 4 shows the sensitivity and specificity of ROC-derived cut-offs for TAPSE and TV S' in SAE prediction.

Multivariable analysis testing all clinical and echocardiographic parameters which were found to be significant in the univariable analysis were

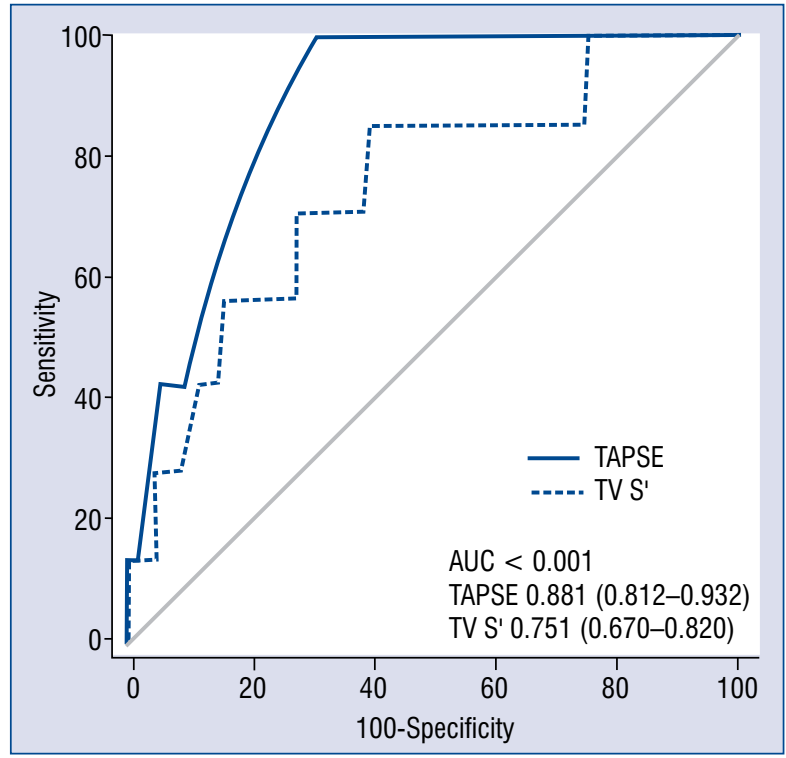

Figure 3. Receiver-operating characteristic (ROC) analysis of tricuspid annular plane systolic excursion (TAPSE) and peak systolic velocity of the lateral part of tricuspid annulus (TV $\mathrm{S}^{\prime}$ ) in serious adverse event prediction in 139 normotensive pulmonary embolism patients. ROC analysis of TAPSE and TV S' for 30-day pulmonary embolism-related mortality and/or need for rescue thrombolysis in normotensive patients; AUC - area under the curve.

performed. Multivariable analysis showed that the optimal prediction model included TAPSE and systemic SBP only, while other clinical and echocardiographic parameters, with TV S' were not included. SBP showed HR 0.89 95\% CI 0.83-0.95, $\mathrm{p}<0.001$ and TAPSE HR $0.67,95 \%$ CI $0.52-0.87$, $\mathrm{p}<0.03$. Kaplan-Meier analysis showed that initially PE patients with TAPSE $\geq 18 \mathrm{~mm}$ had much more favorable prognosis that patients with TAPSE $<18 \mathrm{~mm}$ $(\mathrm{p}<0.01)$, while Kaplan-Meier analysis of S' was only of borderline statistical significance (Fig. 4).

\section{Discussion}

Normotensive patients with acute PE include subjects with a benign clinical course, as well as patients with an increased risk of PE-related mortality, who can deteriorate despite anticoagulation. Although short-term prognosis in acute PE predominantly depends on the hemodynamic status, RVD detected at echocardiography has significant prognostic value, especially in initially hemodynamically stable PE patients [5]. Several echocardiographic parameters for quantitative assessment of RV systolic function have been intensively studied, however echocardiographic assessment 


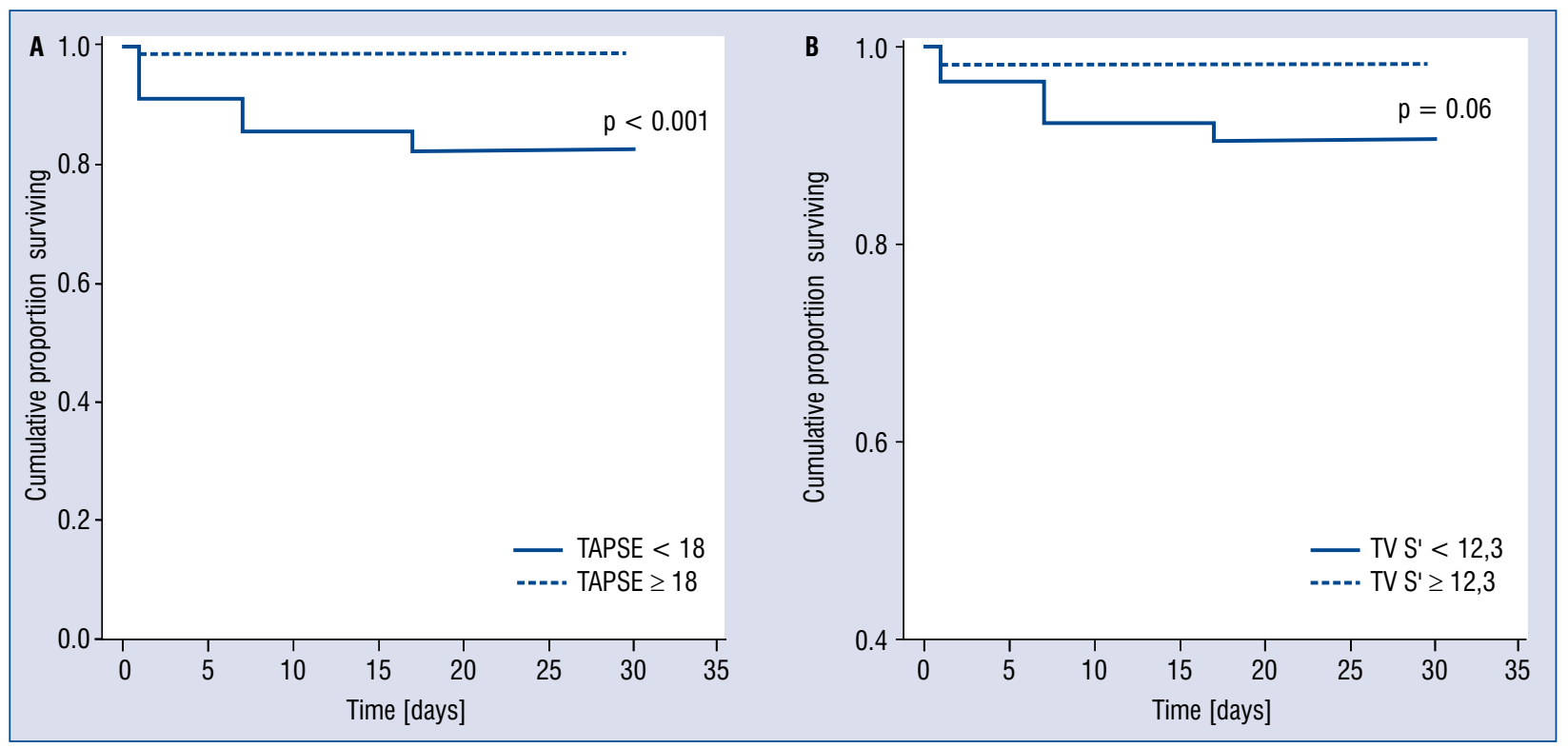

Figure 4. Kaplan-Meier analysis of tricuspid annular plane systolic excursion (TAPSE; A) and peak systolic velocity of lateral part of tricuspid annulus (TV S', B) for event free 30 day survival in 139 initially normotensive patients.

of $\mathrm{RV}$ function remains challenging because of the complex RV anatomy. Various echocardiographic criteria have been proposed as prognostic parameters, however decreased TAPSE was recently reported to be superior to other indices including $\mathrm{RV} / \mathrm{LV}$ ratio or TRPG $[5,6]$.

There is accumulating evidence that tricuspid valve systolic velocity assessed with tissue Doppler is a significant prognostic parameter in various diseases affecting RV [20]. Moreover, the assessment of RVD with tissue Doppler was reported to be related to pulmonary artery thromboembolic burden. Mid-right ventricular myocardial longitudinal dysfunction quantified by tissue Doppler was related to the degree of pulmonary vascular obstruction [21]. Since tissue Doppler has become widely available and the measurement of peak systolic velocity of tricuspid annulus can be performed easily, which were compared herein, the two parameters of RV systolic function, TAPSE or TV S', was more useful in the prognosis assessment in initially hemodynamically stable patients with acute PE. A univariable analysis revealed that both indices significantly predicted a complicated clinical outcome, defined by hemodynamic collapse and need for rescue thrombolysis or PE-related 30-day mortality or both. Additionally, TAPSE and TV S' were characterized by a high AUC in ROC analysis (AUC $0.881 ; 95 \%$ CI $0.812-0.932$ $\mathrm{p}=0.0001$; and AUC $0.751 ; 95 \%$ CI $0.670-0.820$, $\mathrm{p}=0.001$, respectively). Furthermore, Youden's index was used to determine optimal predictive values for both parameters. In the group of 139 consecutive PE patients there were $7 \mathrm{SAE}$ cases including $3 \mathrm{PE}$ related deaths. TV S' $<12.3 \mathrm{~cm} / \mathrm{s}$ was characterized by HR of $4.3,95 \%$ CI $0.8-22.3$, $\mathrm{p}=0.08$ for complicated clinical course and showed $86 \%$ sensitivity and $60 \%$ specificity in SAE prediction. However, when all significant clinical and echocardiographic parameters were included in the multivariable analysis only systemic SBP and TAPSE were found to be of predictive value. Thus, it seems that TV S' is not superior to TAPSE in early risk stratification in normotensive patients with acute PE. The present data corresponds with a recent observation that TAPSE is a reliable predictor of RV systolic dysfunction, and that TAPSE can be recommended for clinical use [22]. Interestingly, no significant differences were found in the initial values of TAPSE or TV S' between patients who died 1 year after discharge and patients who are still alive.

\section{Limitations of the study}

The main limitation of the current study is its single center character with a relatively small number of patients studied and a low number of clinical end points. Therefore, the results of the current study should be interpreted with caution.

Additionally, the DTI measurement is angle-dependent and both TAPSE and TV S' measurements are influenced by movement of the whole heart. 


\section{Conclusions}

Although TV S' predicts short term outcome in normotensive patients with acute symptomatic $\mathrm{PE}$, it seems to be inferior to TAPSE for 30 day prediction of adverse outcome and therefore TAPSE should be recommended as part of the echocardiographic assessment in this group of patients.

\section{Acknowledgements}

The authors wish to thank Dominika Dulny, the Echolab nurse for preparing echocardiographic examinations for the analysis.

\section{Conflict of interest: None declared}

\section{References}

1. Konstantinides SV, Torbicki A, Agnelli G, et al. 2014 ESC guidelines on the diagnosis and management of acute pulmonary embolism. Eur Heart J. 2014; 35(43): 3033-3069, doi: 10.1093/ eurheartj/ehu283, indexed in Pubmed: 25173341.

2. Grifoni S, Olivotto I, Cecchini P, et al. Short-term clinical outcome of patients with acute pulmonary embolism, normal blood pressure, and echocardiographic right ventricular dysfunction. Circulation. 2000; 101(24): 2817-2822, indexed in Pubmed: 10859287.

3. Jaff MR, McMurtry MS, Archer SL, et al. Management of massive and submassive pulmonary embolism, iliofemoral deep vein thrombosis, and chronic thromboembolic pulmonary hypertension: a scientific statement from the American Heart Association. Circulation. 2011; 123(16): 1788-1830, doi: 10.1161/ CIR.0b013e318214914f, indexed in Pubmed: 21422387.

4. Becattini C, Agnelli G, Vedovati MC, et al. Multidetector computed tomography for acute pulmonary embolism: diagnosis and risk stratification in a single test. Eur Heart J. 2011; 32(13): 1657-1663, doi: 10.1093/eurheartj/ehr108, indexed in Pubmed: 21504936.

5. Pruszczyk P, Goliszek S, Lichodziejewska B, et al. Prognostic value of echocardiography in normotensive patients with acute pulmonary embolism. JACC: Cardiovascular Imaging. 2014; 7(6): 553-560, doi: 10.1016/j.jcmg.2013.11.004.

6. Lobo JL, Holley A, Tapson V, et al. Prognostic significance of tricuspid annular displacement in normotensive patients with acute symptomatic pulmonary embolism. J Thromb Haemost. 2014; 12(7): 1020-1027, doi: $10.1111 /$ jth.12589, indexed in Pubmed: 24766779.

7. Saxena N, Rajagopalan N, Edelman K, et al. Tricuspid annular systolic velocity: a useful measurement in determining right ventricular systolic function regardless of pulmonary artery pressures. Echocardiography. 2006; 23(9): 750-755, doi: 10.1111/j.15408175.2006.00305.x, indexed in Pubmed: 16999693.

8. Kim H, Jung C, Yoon HJ, et al. Prognostic value of tricuspid annular tissue Doppler velocity in heart failure with atrial fibrillation. J Am Soc Echocardiogr. 2012; 25(4): 436-443, doi: 10.1016/j. echo.2011.12.019, indexed in Pubmed: 22245051.

9. Kwon DA, Park JS, Chang HJ, et al. Prediction of outcome in patients undergoing surgery for severe tricuspid regurgitation following mitral valve surgery and role of tricuspid annular systolic velocity. Am J Cardiol. 2006; 98(5): 659-661, doi: 10.1016/j. amjcard.2006.03.047, indexed in Pubmed: 16923456.
10. Rudski LG, Lai WW, Afilalo J, et al. Guidelines for the echocardiographic assessment of the right heart in adults: a report from the American Society of Echocardiography endorsed by the European Association of Echocardiography, a registered branch of the European Society of Cardiology, and the Canadian Society of Echocardiography. J Am Soc Echocardiogr. 2010; 23(7): 685-713; quiz 786, doi: 10.1016/j.echo.2010.05.010, indexed in Pubmed: 20620859.

11. Lang R, Badano L, Mor-Avi V, et al. Recommendations for Cardiac Chamber Quantification by Echocardiography in Adults: An Update from the American Society of Echocardiography and the European Association of Cardiovascular Imaging. Eur Heart J Cardiovasc Imaging. 2015; 16(3): 233-271, doi: 10.1093/ehjci/ jev014.

12. Meluzín J, Spinarová L, Bakala J, et al. Pulsed Doppler tissue imaging of the velocity of tricuspid annular systolic motion; a new, rapid, and non-invasive method of evaluating right ventricular systolic function. Eur Heart J. 2001; 22(4): 340-348, doi: 10.1053/euhj.2000.2296, indexed in Pubmed: 11161953.

13. Rodrigues AC, Cordovil A, Monaco C, et al. Right ventricular assessment by tissue-Doppler echocardiography in acute pulmonary embolism. Arq Bras Cardiol. 2013; 100(6): 524-530, doi: 10.5935/abc.20130099, indexed in Pubmed: 23657266.

14. Park JH, Kim JH, Lee JH, et al. Evaluation of right ventricular systolic function by the analysis of tricuspid annular motion in patients with acute pulmonary embolism. J Cardiovasc Ultrasound. 2012; 20(4): 181-188, doi: 10.4250/jcu.2012.20.4.181, indexed in Pubmed: 23346287.

15. Rydman R, Larsen F, Caidahl K, et al. Right ventricular function in patients with pulmonary embolism: early and late findings using Doppler tissue imaging. J Am Soc Echocardiogr. 2010; 23(5): 531-537, doi: 10.1016/j.echo.2010.03.002, indexed in Pubmed: 20381312.

16. Rodrigues AC, Cordovil A, Mônaco CG, et al. Assessing prognosis of pulmonary embolism using tissue-Doppler echocardiography and brain natriuretic peptide. Einstein (Sao Paulo). 2013; 11(3): 338-344, indexed in Pubmed: 24136761.

17. Petkov R, Yamakova Y, Petkova E. Prognostic value of TDI assessment of right ventricular function in patients with acute pulmonary embolism. Eur Respir J. 2014; 44: P2303.

18. Kurnicka K, Lichodziejewska B, Goliszek S, et al. Echocardiographic Pattern of Acute Pulmonary Embolism: Analysis of 511 Consecutive Patients. J Am Soc Echocardiogr. 2016; 29(9): 907-913, doi: 10.1016/j.echo.2016.05.016, indexed in Pubmed: 27427291.

19. McConnell MV, Solomon SD, Rayan ME, et al. Regional right ventricular dysfunction detected by echocardiography in acute pulmonary embolism. Am J Cardiol. 1996; 78(4): 469-473, indexed in Pubmed: 8752195.

20. Smarz K, Zaborska B, Jaxa-Chamiec T, et al. Right ventricular systolic function as a marker of prognosis after ST-elevation inferior myocardial infarction 5-year follow-up. Int J Cardiol. 2016; 221: 549-553, doi: 10.1016/j.ijcard.2016.07.088, indexed in Pubmed: 27414738.

21. Kjaergaard J, Schaadt BK, Lund JO, et al. Quantification of right ventricular function in acute pulmonary embolism: relation to extent of pulmonary perfusion defects. Eur J Echocardiogr. 2008; 9(5): 641-645, doi: 10.1093/ejechocard/jen033, indexed in Pubmed: 18296399.

22. Hamilton-Craig CR, Stedman K, Maxwell R, et al. Accuracy of quantitative echocardiographic measures of right ventricular function as compared to cardiovascular magnetic resonance. Int J Cardiol Heart Vasc. 2016; 12: 38-44, doi: 10.1016/j.ijcha.2016.05.007, indexed in Pubmed: 28616541. 